Pacific Journal of Mathematics

ON THE OPEN CONTINUOUS IMAGES OF PARACOMPACT J J OH MAYS WORked JR. 


\title{
ON THE OPEN CONTINUOUS IMAGES OF PARACOMPACT ČECH COMPLETE SPACES
}

\author{
H. H. WICKE AND J. M. WORRELL, JR.
}

This article characterizes the completely regular $T_{0}$ open continuous images of paracompact Čech complete spaces. The characterization involves three conditions equivalent to being such an image. The first is an intrinsic condition concerning the position of the space in any of its Hausdorff bicompactifications. This condition weakens the condition of Čech completeness by replacing the concept of $G_{\delta}$-set by that of set of interior condensation. This replacement yields a notion of topological completeness which has certain advantages over Cech completeness and uniform completeness but which reduces to Cech completeness in the case of metrizable spaces. The second condition (Condition $\mathscr{C}$ ) is intrinsically defined with the use of a sequence of collections of open sets. It is an analogue of the notion of a regular $T_{0}$-space having a monotonically complete base of countable order. The third condition is that of being an open continuous image of a space which is the sum of open Čech complete subspaces. The main theorem thus displays four equivalent forms of a topological completeness property invariant under open continuous mappings between Tychonoff spaces.

The characterization mentioned (Theorem 4) complements the characterization [15] of the Hausdorif open continuous images of $T_{2}$ paracompact $p$-spaces as Hausdorff spaces of point-countable type in a way analogous to that in which the characterization [14] of regular $T_{0}$ open continuous images of complete metric spaces as regular $T_{0}$ spaces having monotonically complete bases of countable order complements Ponomarev's characterization [11] of the $T_{0}$ open continuous images of metrizable spaces as the $T_{0}$ first countable spaces. It is relevant to recall in this connection some results of Frolik [7] and Arhangel'skii [4], respectively: The class of $T_{2}$ paracompact Čech complete spaces ( $T_{2}$ paracompact $p$-spaces) is the class of $T_{2}$ perfect preimages of complete metric spaces (metric spaces).

In [13] it was shown that a Tychonoff open continuous image of a paracompact Čech complete space (in fact, of a metrically topologically complete space) is not necessarily Čech complete. This affords interesting contrast with the behavior guaranteed by Theorem 5: A Tychonoff open continuous image of a Tychonoff space complete in the sense of Condition $\mathscr{Y}$ is also complete in the same sense.

Some results of the paper have antecedents in the classical theory 
of metrically topologically complete spaces (i.e., spaces which are homeomorphic to complete metric spaces). Particularly relevant is the theorem of Cech [6] that a metrizable space is topologically complete if and only if it is a $G_{\delta}$-subset of its Stone-Čech bicompactification. Theorem 4 provides an analogue of this in which the concept of metric topological completeness is replaced by Condition $\mathscr{K}$ and the concept of $G_{i}$-set is replaced by that of a set of interior condensation. Certain results on the invariance of $G_{\tilde{o}}$-sets in Euclidean space under open continuous mappings also are relevant; they are referred to after Theorem 2.

The plan of exposition is to introduce appropriate concepts and prove three theorems concerning these concepts from which Theorem 4 readily follows. The first of these theorems gives a sufficient condition that a regular $T_{0}$-space be an open continuous image of a paracompact Čech complete space. The second theorem concerns the behavior of certain sets of interior condensation under open continuous mappings and the third one shows that the extrinsic condition mentioned implies the intrinsic condition.

2. Terminology. The terminology generally used here is much like that of [8] except that the null set convention is not used, i.e., all sets herein have elements. If $A$ and $B$ are sets, $A \cdot B$ denotes the intersection of $A$ and $B$ and $A+B$ denotes the sum or union of $A$ and $B$. Spaces called compact in [8] are here called bicompact following the usage of [1]. The usage of compast spase here is that of Fréchet, namely, that there exists no infinite subset of the space which does not have a limit point. If $K$ is a collection of sets, $K^{*}$ denotes the sum of the members of $K$. The term inner limiting set is synonymous with $G_{\delta}$-set. A Tychonoff space $S$ (三 completely regular $T_{0}$-space) is said to be Cech complete [6] if and only if it is an inner limiting set in its Stone-Čech bicompactification. A space is said to be metrically topologically complete if and only if it has a topology-preserving metric in which it is complete. The letters $i, j, k$, and $n$ are used to signify positive integers.

3. Condition $\mathscr{r}$. A subset $M$ of a topological space $(S, \tau)$ is said to be of countable character in $S$ [5] if and only if there exists a sequence $D_{1}, D_{2}, \cdots$ of elements of $\tau$ including $M$ such that any member of $\tau$ which includes $M$ also includes some $D_{n}$.

A space $S$ is said to be of point-countable type [5] if and only if $S$ is covered by a collection of bicompact sets of countable character in $S$.

In [15] it is shown that a Hausdorff space is of point-countable type if and only if it is an open continuous image of a Hausdorff paracompact $p$-space. All first-countable spaces and all Tychonoff $p$ spaces are of point-countable type and the property of being of point- 
countable type is preserved by open continuous mappings [5].

LEMMA 1 [15]. In a Hausdorff spase $S$ the following properties are equivalent:

(i) $S$ is of point-countable type.

(ii) If $D$ is open in $S$ and $P$ is in $D$ there exists a bicompact set $\beta$ of countable character which contains $P$ and is a subset of $D$.

Definition. A topological space $(S, \tau)$ is said to satisfy Condition $\mathscr{K}$ if and only if there exists a sequence $G_{1}, G_{2}, \cdots$ of subcollections of $\tau$ covering $S$ such that: (1) For each $n$, if $P$ belongs to an element $g$ of $G_{n}$, there exists a member $g^{\prime}$ of $G_{n+1}$ containing $P$ such that $g$ includes $\bar{g}^{\prime}$. (2) If $g_{1}, g_{2}, \cdots$ is a sequence such that, for each $n, g_{n}$ is a member of $G_{n}$ including $\overline{g_{n+1}}$, then there exists a (nonempty) bicompact set $\beta$ which is the common part of the terms of $g_{1}, g_{2}, \cdots$ such that any member of $\tau$ which includes $\beta$ also includes some $g_{n}$.

Spaces satisfying Condition $\mathscr{K}$ are of point-countable type. Thus in the Hausdorif case Lemma 1 applies to such spaces. This remark is used in the proof of Theorem 1.

Theorem 1 is a major component of Theorem 4. It is related to the theorem of [15] stated above and also to other theorems regarding the existence of open mappings $[2,11,14,16]$. Recall that the weight of a topological space is the smallest cardinal number $m$ such that the space has a base of cardinal $m$ [1].

Theorem 1. Suppose $S$ is a regular $T_{0}$-spase. If $S$ satisfies Condition $\mathscr{K}$ then $S$ is an open continuous image of a parasompact Cech complete space of the same weight as $S$.

Proof. Let $\tau$ denote the topology of $S$. Since $S$ satisfies Condition $\mathscr{K}$, it may be seen that there exists a sequence $H_{1}, H_{2}, \ldots$ of well-ordered subcollections of $\tau$ covering $S$ such that these conditions are satisfied: (1) For each $n$, each $h$ in $H_{n}$ contains a point $X_{n, h}$ belonging to no preceding element of $H_{n}$. (2) If $n<k$ and $P$ is a point of $S$, the closure of the first element of $H_{k}$ containing $P$ is a subset of the first element of $H_{n}$ containing $P$. (3) If $h_{1}, h_{2}, \cdots$ is a sequence such that each $h_{n}$ belongs to $H_{n}$ and each $h_{n}$ is the first element of $H_{n}$ containing $X_{n+1, h_{n+1}}$, then there exists a bicompact point set $\beta$ such that (a) $\beta$ is the common part of the terms of $h_{1}, h_{2}, \ldots$ and (b) every element of $\tau$ which includes $\beta$ also includes some $h_{n}$.

Let $\Gamma$ denote the collection of all bicompact point sets $\gamma$ of $(S, \tau)$ such that for some decreasingly monotonic sequence $D_{1, \gamma}, D_{2, \gamma}, \cdots$ of elements of $\tau$ including $\gamma$ it is true that every element of $\tau$ in which $\gamma$ lies includes some $D_{n, r}$. Let $L_{S}$ denote the weight of $S$. Let $\tau^{\prime}$ denote a base for $S$ such that $\overline{\bar{\tau}}^{\prime}=L_{S}$. 
There exists a meaning for the notation $U_{n, \omega}$ for positive integers $n$ and certain ordered pairs $\omega$, such that with respect to some sequences $\Omega_{1}, \Omega_{2}, \cdots$ and $Q_{2}, Q_{3}, \cdots$ of transformations these conditions are satisfied: (1) For each $n$, the range of $\Omega_{n}$ is a subcollection of $\tau$ covering $S$. (2) For each $n>1, \Omega_{n}$ and $\Omega_{n-1}$ are, respectively, the domain and range of $Q_{n}$. (3) If for each $n$ and $\omega$ in $\Omega_{n}, B_{\omega}$ denotes the second term of $\omega$, then for each $n>1$ and $\omega$ in $\Omega_{n-1}, P$ belongs to $B_{\omega}$ if and only if $P$ belongs to the second term of some member of $Q_{n}^{-1}(\omega)$. (4) For each $n, \bar{\Omega}_{n} \leqq L_{S}+\boldsymbol{\aleph}_{0}$. (5) For each $n$ and $\omega$ in $\Omega_{n}, U_{n, \omega}$ is a finite sub-collection of $\tau$ covering $B_{\omega}$ such that each $u$ in $U_{n, \omega}$ contains a point $Y_{n, \omega, u}$ such that $\bar{u}$ is a subset of the first element of $H_{n}$ that contains $Y_{n, \omega, u}$. (6) For each $n>1$ and $\omega$ in $\Omega_{n-1}$, if $\omega^{\prime}$ belongs to $Q_{n}^{-1}(\omega)$ and $u$ belongs to $U_{n, \omega^{\prime}}$, then there exists an element $u^{\prime}$ of $U_{n-1, \omega}$ such that $B_{\omega} \cdot u^{\prime}$ includes $\bar{u}$. (7) If $\gamma$ belongs to $\Gamma$ there exists some $\omega$ in $\Omega_{1}$ such that $B_{\omega}$ includes $\gamma$ and $D_{1, r}$ includes $B_{\omega}$. Moreover, if $n>1$, and $\omega$ belongs to $\Omega_{n-1}$, and $\gamma$ is a subset of $B_{\omega}$ belonging to $\Gamma$ there exists some $\omega^{\prime}$ in $Q_{n}^{-1}(\omega)$ such that $B_{\omega^{\prime}}$ includes $\gamma$ and $D_{n, r}$ includes $U_{n, \omega^{\prime}}^{*}$ (This construction may be carried out using (7) as a starting point.)

Let $E$ denote the set of all sequences $\left(\omega_{1}, P\right),\left(\omega_{2}, P\right), \cdots$ such that (1) each $\omega_{n}$ belongs to $\Omega_{n}$, (2) each $\omega_{n}$ is $Q_{n+1}\left(\omega_{n+1}\right)$, and (3) each $B_{\omega_{n}}$ contains $P$. Let $\psi$ denote the collection to which an element belongs if and only if it is the sum of some sets $D$, such that for some $n$, some $\omega$ in $\Omega_{n}$, and some subset $U$ of $B_{\omega}$ belonging to $\tau, D$ is the set of all sequences $\left(\omega_{1}, P\right),\left(\omega_{2}, P\right), \cdots$ in $E$ such that $\omega=\omega_{n}$ and $U$ contains $P$. Let $\varphi$ denote the transformation of $E$ such that for each sequence $\left(\omega_{1}, P\right),\left(\omega_{2}, P\right), \cdots$ belonging to $E, \varphi\left[\left(\omega_{1}, P\right),\left(\omega_{2}, P\right), \cdots\right]=P$.

It may be shown that $(E, \psi)$ is a regular $T_{0}$ topological space, that $\varphi$ maps $(E, \psi)$ onto $(S, \tau)$ continuously, and that $\varphi(D)$ belongs to $\tau$ for each $D$ in $\psi$. If it can be shown that $(E, \psi)$ has a metrically topologically complete upper semicontinuous decomposition into bicompact sets, it follows from Frolik's theorem [7] that $(E, \psi)$ is a paracompact C̈ech complete space.

For each $n$ and $\omega$ in $\Omega_{n}$, let $t_{n, \omega}$ denote the set of all sequences $\left(\omega_{1}, P\right),\left(\omega_{2}, P\right), \cdots$ in $E$ such that $\omega=\omega_{n}$ and $B_{\omega}$ contains $P$. Let $V_{n}$ denote the collection of all these sets $t_{n, \omega}$. Let $G$ denote the collection to which $g$ belongs if and only if there exists a decreasingly monotonic sequence $v_{1}, v_{2}, \cdots$ of sets such that each $V_{n}$ contains $v_{n}$ and $g$ is the common part of the sets $v_{n}$. It may be shown that there exists a transformation $\theta$ of $G$ such that if $(1) \omega_{1}, \omega_{2}, \cdots$ is a sequence such that each $\omega_{n}$ belongs to $\Omega_{n}$ and each $\omega_{n}$ is $Q_{n+1}\left(\omega_{n+1}\right)$ and (2) if $g$ is the common part of the sets $t_{n, \omega_{n}}$, then $\theta(g)$ is $B_{\omega_{1}} \cdot B_{\omega_{2}} \cdots$. Moreover, it may be seen that if $g$ belongs to $G, \theta(g)=\varphi(g)$ and $\varphi \mid g$ is reversibly continuous.

Suppose that $\omega_{1}, \omega_{2}, \cdots$ is a sequence such that each $\omega_{n}$ belongs 
to $\Omega_{n}$ and each $\omega_{n}$ is $Q_{n+1}\left(\omega_{n+1}\right)$. (A) If $u_{1}, u_{2}, \cdots$ is a sequence such that each $u_{n}$ belongs to $U_{n, \omega_{n}}$ and each $u_{n}$ includes $\overline{u_{n+1}}$ then for each $n$ there exists a first element $h_{n}$ of $H_{n}$ that includes some term of $u_{1}, u_{2}, \cdots$. If $n<k$ there exists some $i>k$ such that $u_{i}$ is a subset of $h_{n}$ and of $h_{k}$. Moreover, $u_{i}$ is a subset of the first element $h$ of $H_{n}$ containing $Y_{i, \omega_{i}, u_{i}}$. So $h$ does not precede $h_{n}$. Since $h_{n}$ contains $Y_{i, \omega_{i}, u_{i}}, h$ does not follow $h_{n}$. Hence $h=h_{n}$. Similarly, $h_{k}$ is the first element of $H_{k}$ containing $Y_{i, \omega_{i}, u_{i}}$. So $h_{n}$ includes $h_{k}$. Thus $h_{n}$ is the first element of $H_{n}$ containing $X_{k, h_{k}}$. So each $h_{n}$ includes $\overline{h_{n+1}}$ and there exists a bicompact point set $\beta$ such that $\beta$ is the common part of the sets $h_{n}$ and every element of $\tau$ in which $\beta$ lies includes some $h_{n}$. Hence each $\bar{u}_{n}$ intersects $\beta$. Moreover, $u_{1} \cdot u_{2} \cdot \ldots$ is a bicompact subset $\beta^{\prime}$ of $\beta$. If $D$ is a member of $\tau$ in which $\beta^{\prime}$ lies but which includes none of the sets $u_{n}$, there exists a sequence $P_{1}, P_{2}, \cdots$ of distinct points of $S$ such that each $\left[u_{n}-\left(D \cdot u_{n}\right)\right] \cdot h_{n}$ contains $P_{n}$. But a contradiction is involved, for $\left\{P_{1}\right\}+\left\{P_{2}\right\}+\cdots$ has a limit point $X$ belonging to $\beta$, and each $\bar{u}_{n}$ contains $X$, and each $u_{n}$ includes $\overline{u_{n+1}}$. (B) Since each $B_{\omega_{n-1}}$ includes $U_{n, \omega_{n}}^{*}$, the terms of the sequence $B_{w_{1}}$, $B_{\omega_{2}}, \cdots$ have a common part $M$. Since each $B_{\omega_{n}}$ includes $\overline{B_{\omega_{n+1}}}, M$ is closed. If $J$ is an infinite subset of $M$ then, since each of the finite collections $U_{n, \omega_{n}}$ covers $B_{\omega_{n}}$, each of the collections has an element having an infinite intersection with $J$. With application of Konig's lemma it may be seen that there exists a sequence $u_{1}, u_{2}, \cdots$ as in (A) such that each $u_{n}$ contains infinitely many points of $J$. Since every member of $\tau$ in which the bicompact set $u_{1} \cdot u_{2} \ldots$ lies includes some $u_{n}, J$ has a limit point belonging to $u_{1} u_{2} \cdots$ (C) Let $B$ denote the collection of all sets $\beta^{\prime}$ as in (A). If $W$ is a subcollection of $\psi$ covering $M$ then for each $\beta$ in $B$ there exists a finite subcollection $T_{\beta}$ of $W$ covering $\beta$. For each $\beta$ there exists some positive integer $n$ such that some element $u$ of $U_{n, \omega_{n}}$ including $\beta$ is a subset of $T_{\beta}^{*}$. Since $U_{1, \omega_{1}}+U_{2, \omega_{2}}+\cdots$ is countable, there exists a sequence $\beta_{1}, \beta_{2}, \cdots$ of elements of $B$ such that $T_{\beta_{1}}+T_{\beta_{2}}+\cdots$ covers $M$. Thus $M$ is Lindelofian. Since $M$ is $T_{1}$ and compact, $M$ is bicompact. For reasons similar to some involved in $(A)$, every member of $\tau$ in which $M$ lies includes some $B_{\omega_{n}}$. $(D)$ Let $g$ denote $t_{1, \omega_{1}}, t_{2, \omega_{2}} \cdots$. Since $\varphi \mid g$ is reversibly continuous, and $\varphi(g)=M, g$ is a $T_{2}$ bicompact point set. With the use of $(C)$ it may be shown that every element of $\psi$ 's in which $g$ lies includes some $t_{n, \omega_{n}}$. Moreover, for each $n$ every member of $V_{n}$ includes every element of $G$ that it intersects. Thus $G$ is upper semicontinuous. $(E)$ With straightforward application of the conditions on $\Omega_{1}, \Omega_{2}, \cdots$ and $Q_{2}, Q_{3}, \cdots$ it may be seen that if $\gamma$ belongs to $\Gamma$ there exists a sequence $\omega_{1}, \omega_{2}, \cdots$ as above such that $\gamma$ is a point set $M$ as in $(B)$. Moreover, for each $n$ and $\omega$ in $\Omega_{n}$, if $\gamma$ is a 
subset of $B_{\omega}$ belonging to $\Gamma$ then there exists such a sequence $\omega_{1}$, $\omega_{2}, \cdots$ such that $\omega_{n}=\omega$ and $\gamma=M$.

For each $n$, let $\Delta_{n}$ denote the collection to which $\delta$ belongs if and only if for some $v$ in $V_{n}, \delta$ is the collection of all elements of $G$ that intersect $v$. It may be seen that $\Delta_{1}+\Delta_{2}+\cdots$ is a $\sigma$-discrete base for $G$ with respect to the (appropriate) quotient topology $\xi$. Thus, since $(G, \xi)$ is a regular $T_{0}$-space, $(G, \xi)$ is metrizable. With use of the above paragraphs it may be seen that if $D_{1}, D_{2}, \ldots$ is a decreasingly monotonic sequence such that each $D_{n}$ belongs to $\Delta_{n}+\Delta_{n+1}+\cdots$, then $\left\{D_{1}\right\}+\left\{D_{2}\right\}+\cdots$ is a base for $(G, \xi)$ at some point. It follows that $(G, \xi)$ is metrically topologically complete. With the use of condition (4) on $\Omega_{1}, \Omega_{2}, \cdots$ and $Q_{2}, Q_{3}, \cdots$, it may be shown that if $\boldsymbol{K}$ is the weight of $(E, \psi)$ then $\boldsymbol{\aleph}+\boldsymbol{\aleph}_{0}=L_{S}+\boldsymbol{\aleph}_{0}$.

4. Sets of interior condensation. In this section we introduce a concept which has been essential in the authors' discussions of topological completeness in structures more general than metrizable spaces $[17,18]$. The concept plays a role in these more general settings comparable to that played by inner limiting sets in the theory of Moore spaces and metrizable spaces.

Suppose $S$ is a subset of a set $E$. A sequence $G_{1}, G_{2}, \cdots$ of collections of subsets of $E$ covering $S$ is said to be a monotonically contrasting sequense of $S$ in $E$ if and only if, for each $n$, if $P$ is an element of $S$ belonging to a member $g$ of $G_{n}$, there exists a subset of $g$ which contains $P$ and belongs to $G_{n+1}$.

A subset $S$ of a topological space $E$ is said to be a set of interior condensation in $E$ if and only if there exists a monotonically contracting sequence $G_{1}, G_{2}, \cdots$ of $S$ in $E$ such that (1) each element of each $G_{n}$ is open in $E$, and (2) if $P$ is a point belonging to each term of a sequence $g_{1}, g_{2}, \cdots$ such that, for each $n, g_{n}$ belongs to $G_{n}$ and includes $g_{n+1}$, then $P$ belongs to $S$.

It is clear that inner limiting sets are sets of interior condensation. It may be shown that sets of interior condensation in Moore spaces are inner limiting sets in these spaces.

The proof of the next theorem demonstrates the invariance of the property of being an absolute set of interior condensation under open mappings between Tychonoff spaces. This theorem shows the advantage obtained by relaxing the condition of being an inner limiting set to that of being a set of interior condensation, for there exists an open mapping between Tychonoff spaces whose domain is an absolute inner limiting set (Čech complete) and whose range is not. Such an example may be obtained by applying Theorem 2 of [14] to any non-Cechcomplete subspace of the space $\Omega$ of countable ordinals with the order 
topology [13]. (Every subspace of $\Omega$ is regular, $T_{0}$, and has a monotonically complete base of countable order and is therefore an open continuous image of a complete metric space.)

Recall first that a mapping $\phi$ of a space $S$ onto a space $R$ is said to be inductively open [3] if and only if there exists a subspace $S^{\prime}$ of $S$ such that $\varphi \mid S^{\prime}$ is open and $\varphi\left(S^{\prime}\right)=R$.

THEOREM 2. Suppose $S$ is a set of interior condensation in a $T_{2}$ bicompact space. Then any inductively open continuous image of $S$ is a set of interior condensation in any $T_{2}$-space of which it is a dense subspace.

Proof. Let $(E, \psi)$ denote a $T_{2}$ bicompact space of which $(S, \tau)$ is a subspace. Let $G_{1}, G_{2}, \cdots$ denote a sequence of subcollections of $\psi$ covering $S$ which satisfies the conditions of the definition of set of interior condensation. Let $\varphi$ denote a continuous mapping of $S$ onto a space $R$ and $S^{\prime}$ a subspace of $S$ such that $\varphi \mid S^{\prime}$ is open and $\varphi\left(S^{\prime}\right)=$ $R$. Let $(F, \sigma)$ denote a $T_{2}$-space of which $R$ is a dense subspace. There exists a sequence $H_{1}, H_{2}, \cdots$ of well-ordered subcollections of $\psi$ covering $S$ such that these conditions are satisfied for each $n$ : (S1) Each $h$ in $H_{n}$ contains a point of $S$ not in any predecessor of $h$. (S2) If $P$ is in $S, n<k$, and $h$ and $h^{\prime}$ are the first elements of $H_{n}$ and $H_{k}$ that contain $P$, respectively, then $h$ includes $h^{\prime}$. (S3) If $P$ is in $E$ and $h_{1}, h_{2}, \cdots$ is a sequence such that each $h_{n}$ is a member of $H_{n}$ which includes $h_{n+1}$ and contains $P$, then $P$ belongs to $S$. By using a technique related to one used in the proof of the Lemma on page 261 of [16] it may be shown that there exists a sequence $W_{1}, W_{2}, \ldots$ of well-ordered subcollections of $\sigma$ covering $R$ such that these conditions are satisfied for each $n$ : (W1) Each $w$ in $W_{n}$ contains a point of $R$ not in any predecessor of $w$. (W2) If $P$ is in $R, n<k$, and $w$ and $w^{\prime}$ are the first elements of $W_{n}$ and $W_{k}$, respectively, that contain $P$, then $w$ includes $w^{\prime}$. There also exists a function $D$ such that for each $n$ : (D1) If $w$ is in $W_{n}, D_{n, w}$ is an element of $\psi$ such that

$$
\varphi\left(D_{n, w} \cdot S^{\prime}\right)=w \cdot R \text {. }
$$

(D2) If $P$ is in $R, n<k$, and $w$ and $w^{\prime}$ are the first elements of $W_{n}$ and $W_{k}$, respectively, that contain $P$, then $\bar{D}_{k, w^{\prime}}^{E}$ is a subset of $D_{n, w}$. (D3) If $w$ is in $W_{n}$, there exists a point $X$ of $D_{n, w} \cdot S^{\prime}$ such that the first element of $H_{n}$ that contains $X$ includes $D_{n, w}$.

Let $V_{1}$ denote $W_{1}$ and for each $n>1$ let $V_{n}$ denote the collection of all sets of the form $v \cdot w$ where $w$ is in $W_{n}$ and $v$ belongs to $V_{n-1}$ and contains a point of $w$ not in any predecessor of $w$ in $W_{n}$. Then $V_{1}, V_{2}, \cdots$ is a sequence of subcollections of $\sigma$ covering $R$. If $P$ is 
in the element $v$ of $V_{n}$ and $w$ is the first element of $W_{n+1}$ that contains $P$, then $v \cdot w$ is a subset of $v$ which contains $P$ and belongs to $V_{n+1}$. Suppose $P$ is a point belonging to each term of a sequence $v_{1}, v_{2}, \ldots$ where each $v_{n}$ belongs to $V_{n}$ and includes $v_{n+1}$. For each $n$ there exists a first $w_{n}$ in $W_{n}$ that includes a term of $v_{1}, v_{2}, \cdots$. For each $n$ there exists $j>n+1$ such that $v_{j}$ is a subset of $w_{n}$ and $w_{n+1}$. The set $v_{j}$ is of the form $v \cdot w$ where $v$ is in $V_{j-1}, w$ is in $W_{j}$, and $v$ contains a point $X$ of $w$ not in any predecessor of $w$. Let $h$ denote the first element of $W_{n}$ that contains $X$. Then $h$ includes $v \cdot w$. Hence $w_{n}$ does not follow $h$. Since $w_{n}$ contains $X$ it follows that $w_{n}=h$. Similarly $w_{n+1}$ is the first element of $W_{n+1}$ that contains $X$. Hence $w_{n}$ includes $w_{n+1}$ and, letting $D_{n}$ denote $D_{n, w_{n}}$, it follows that $\bar{D}_{n+1}^{E}$ is a subset of $D_{n}$. Since $E$ is bicompact, it follows that the sets $D_{1}, D_{2}, \cdots$ have a (nonempty) closed and bicompact common part $K$. By condition (D3) for each $n$ there exists a first $h_{n}$ in $H_{n}$ that includes a term of $D_{1}, D_{2}, \cdots$. By (D3) and an argument similar to one used just above it follows that, for each $n, h_{n}$ includes $h_{n+1}$. Since $K$ is a subset of each $h_{n}$ it follows from (\$3) that $K$ is a subset of $S$. Suppose $P$ does not belong to $\varphi(K)$. Since $\varphi(K)$ is bicompact and $F$ is $T_{2}, \varphi(K)$ is closed and there exists an open set $D$ which includes $\varphi(K)$ such that $P$ is not in $\bar{D}$. Since $R$ is dense in $F$, for each $n$, there exists $Y_{n}$ in $R$ such that $Y_{n}$ belongs to $w_{n}-\bar{D}$ and there exists $X_{n}$ in $D_{n}$. $S^{\prime}$ such that $\varphi\left(X_{n}\right)=Y_{n}$. There exists a point $Z$ such that $Z$ is either a limit point of the set of all $X_{n}$ 's or else $X_{n}=Z$ for infinitely many $n$. In either case $Z$ belongs to $K$ so that $\varphi(Z)$ is in $\varphi(K)$ and thus $\varphi(Z)$ is in $D$. Since $\varphi$ is continuous this implies that infinitely many $Y_{n}$ belong to $D$ which involves a contradiction. Therefore $P$ belongs to $\varphi(K)$ and hence to $R$. Thus $R$ is a set of interior condensation in $F$.

Comment. A classical theorem of Sierpiński [12] on the invariance of $G_{\delta}$-sets in Euclidean $n$-space under open continuous mappings may be derived from this theorem. An earlier result is by Mazurkiewicz [10]. See [9, pp. 430-431] for further discussion.

THEOREM 3. Suppose $S$ is a set of interior condensation in some $T_{2}$ bicompast spase. Then $S$ satisfies Condition $\varkappa_{i}$.

Proof. Suppose $S$ is a set of interior condensation in a $T_{2}$ bicompact space $(E, \psi)$. Let $G_{1}, G_{2}, \cdots$ denote a sequence of $\psi$ which satisfies the conditions of the definition of set of interior condensation. As in the proof of Theorem 2 there exists a sequence $H_{1}, H_{2}, \cdots$ of well-ordered subcollections of $\psi$ satisfying conditions (S1)-(S3) for each $n$. For each $n$, let $V_{n}$ denote the collection of all sets of the form 
$h \cdot S$ where $h$ belongs to $H_{n}$. Suppose $h$ and $h^{\prime}$ are in $H_{n}$ and $h \cdot S=$ $h^{\prime} \cdot S$. Since $h$ contains a point $P$ of $S$ not in any predecessor of $h$ and $h^{\prime}$ contains $P$ it follows that $h$ does not follow $h^{\prime}$. Similarly $h^{\prime}$ does not follow $h$, so that $h=h^{\prime}$. Hence each $V_{n}$ may be well ordered by the prescription that $v$ precedes $v^{\prime}$ if and only if $h$ precedes $h^{\prime}$, where $h$ and $h^{\prime}$ are in $H_{n}$ and $v=h \cdot S, v^{\prime}=h^{\prime} \cdot S$. Suppose $P$ is in $S$. Let $h$ denote the first element of $H_{n}$ that contains $P$. Then $h \cdot S$ is the first element of $V_{n}$ that contains $P$. Suppose $P$ is in $S, n<k$, and $v$ and $v^{\prime}$ are the first elements of $V_{n}$ and $V_{k}$, respectively, that contain $P$. Suppose $v=h \cdot S$ and $v^{\prime}=h^{\prime} \cdot S$ where $h$ is in $H_{n}$ and $h^{\prime}$ is in $H_{k}$. Then $h$ and $h^{\prime}$ are the first elements of $H_{n}$ and $H_{k}$, respectively, that contain $P$ so that $h$ includes $\bar{h}^{\prime}$. Hence $v$ includes $\bar{v}^{\prime}$ (the closure of $v^{\prime}$ with respect to $S$ ). Suppose that $v_{1}, v_{2}, \cdots$ is a sequence such that, for each $n, v_{n}$ is an element of $V_{n}$ that includes $\bar{v}_{n+1}^{S}$. For each $n$ there exists a first $h_{n}$ in $H_{n}$ that includes a term of $v_{1}, v_{2}, \cdots$. For each $n$ there exists $j>n+1$ such that $v_{j}$ is a subset of $h_{n}$ and $h_{n+1}$. There exists an $h$ in $H_{j}$ such that $v_{j}=h \cdot S$. Let $X$ denote a point of $h \cdot S$ not in any predecessor of $h$. By an argument used in the proof of Theorem 2 it follows that $h_{n}$ and $h_{n+1}$ are the first elements of $H_{n}$ and $H_{n+1}$, respectively, that contain $X$. It follows that $h_{n}$ includes $\bar{h}_{n+1}$. Hence there exists a bicompact point set $\beta$ which is the common part of the terms of $h_{1}, h_{2}, \ldots$ and which is a subset of $S$. Suppose for some $n, v_{n}$ does not meet $\beta$. Then there exists a sequence $P_{1}, P_{2}, \cdots$ such that, for each $k, P_{k}$ is in $v_{n+k}$ and is not in $\beta$. For each $k$ there exists $n_{k}>k$ such that $P_{n_{k}}$ is in $h_{k}$. If $A$ denotes $\left\{P_{n_{1}}\right\}+\left\{P_{n_{2}}\right\}+\cdots$, then $\bar{A}$ meets $\beta$. For if it does not, some $h_{k}$ does not meet $\bar{A}$. Suppose $P$ is in $\bar{A} \cdot \beta$. Then $P$ is not in $A$ so that every open set containing $P$ contains infinitely many elements of $A$. But then $P$ is in each $\bar{v}_{n}^{S}$ and, therefore, in each $v_{n}$ which is a contradiction. It follows that the common part $\beta^{\prime}$ of the terms of $v_{1}, v_{2}, \cdots$ exists and is a bicompact subset of $\beta$. By an argument used in the proof of Theorem 1 it follows that any open set in $E$ which includes $\beta^{\prime}$ also includes some $v_{n}$.

Let $G_{1}^{\prime}$ denote $V_{1}$. For each $n>1$ let $G_{n}^{\prime}$ denote the collection of all sets of the form $g \cdot v$ where $g$ is an open set of $S, v$ is in $V_{n}, g$ contains a point $P$ of $v$ not in any predecessor of $v$ in $V_{n}$, and for some $g^{\prime}$ in $G_{n-1}^{\prime}, \bar{g}^{s}$ is a subset of $g^{\prime}$. We shall show that $G_{1}^{\prime}, G_{2}^{\prime}, \ldots$ is a sequence enabling $S$ to satisfy Condition $\mathscr{K}$. Suppose $P$ is a point of $g$ in $G_{n}^{\prime}$. There exist a first $v$ in $V_{n+1}$ that contains $P$, and an open set $g^{\prime}$ of $S$ containing $P$ such that $\bar{g}^{\prime S}$ is a subset of $g$. Hence $g^{\prime} \cdot v$ is a member of $G_{n+1}^{\prime}$ which contains $P$ and ${\overline{g^{\prime}} \cdot v}^{S}$ is a subset of $g$. Suppose $g_{1}, g_{2}, \cdots$ is a sequence such that, for each $n, g_{n}$ belongs to $G_{n}^{\prime}$ and includes $\bar{g}_{n+1}^{S}$. For each $n$ there exists a first $v_{n}$ in 
$V_{n}$ that includes a term of $g_{1}, g_{2}, \cdots$. By an argument used in the proof of Theorem 2 it follows that, for each $n, v_{n}$ includes $\bar{v}_{n+1}^{S}$. Thus a bicompact common part $\beta$ of the terms of $v_{1}, v_{2}, \cdots$ exists. By an argument similar to one used in the preceding paragraph it follows that there exists a bicompact point set $\beta^{\prime}$ which is the common part of the terms of $g_{1}, g_{2}, \cdots$ such that any open set in which $\beta^{\prime}$ lies includes some $g_{n}$.

\section{The characterization theorem.}

THEOREM 4. Suppose $S$ is a Tychonoff space. Then the following conditions on $S$ are equivalent:

(a) $S$ is a set of interior condensation in one (equivalently, in each) of its Hausdorff bicompactifications.

(b) $S$ satisfies Condition $\mathscr{K}$.

(c) $S$ is an open continuous image of a paracompact Čech complete space.

(d) $S$ is an open continuous image of a space which is a sum of open Cech complete subspaces.

Proof. Condition (a) implies (b) by Theorem 3. Condition (b) implies (c) by Theorem 1. That (c) implies (d) is obvious. Any space as in (d) is an open continuous image of a Čech complete space. Since a Cech complete space is a set of interior condensation in its StoneČech bicompactification a Tychonoff open continuous image of it is a set of interior condensation in each of its $T_{2}$ bicompactifications by Theorem 2. Thus (d) implies (a).

Comment. The reader is referred to the introduction for a comparison of part of Theorem 4 with a theorem of Čech.

Theorem 5. Suppose $S$ is a Tychonoff space which satisfies Condition $\mathscr{K}$. Then any Tychonoff inductively open continuous image of $S$ satisfies Condition $\mathscr{K}$.

Proof. If $S$ satisfies $\mathscr{K}$ then $S$ is a set of interior condensation in its Stone-Cech bicompactification by Theorem 4. By Theorem 2 any Tychonoff inductively open continuous image of $S$ is a set of interior condensation in its Stone-Čech bicompactification. An application of Theorem 4 completes the proof. 


\section{REFERENCES}

1. P. S. Aleksandrov ( $\equiv$ P. S. Alexandroff) and P. Uryson (=P. Urysohn), Mémoire sur les espaces topologiques compacts, Verh. Nederl. Akad. Wetensch. Afd. Natuurk. Sect. I, 14, no. 1 (1929), 1-96.

2. A. V. Arhangel'skii, On mappings of metric spaces, Dokl. Akad. Nauk SSSR 145 (1962), 245-247 (Russian); Trans. in Soviet Math. Dokl., 3 (1962), 953-956.

3. - On open and almost-open mappings of topological spaces, Dokl. Akad. Nauk SSSR 147 (1962), 999-1002 (Russian).

4. - On a class of spaces containing all metric and all locally bicompact spaces, Mat. Sb., 67 (1965), 55-85 (Russian).

5. — Bicompact sets and the topology of spaces, Trudy Mosk. Mat. Obšč., 13 (1965), 3-55 (Russian).

6. E. Čech, On bicompact spaces, Ann. of Math., 38 (1937), 823-844.

7. Z. Frolik, On the topological product of paracompact spaces, Bull. Acad. Polon. Sci. Sér. Math., Astr., Phys., 8 (1960), 747-750.

8. J. L. Kelley, General Topology, Van Nostrand, Princeton, 1955.

9. C. Kuratowski, Topology Vol. I, New edition, Academic Press, New York, and Warsaw, 1966.

10. S. Mazurkiewicz, Über Borelsche Mengen, Bull. Acad. Cracovie, (1916), 490-494.

11. V. I. Ponomarev, Axioms of countability and continuous mappings, Bull. Acad. Polon. Sci., Sér. Sci. Math., Astr. Phys., 8 (1960), 127-133 (Russian).

12. W. Sierpiński, Sur une propriété des ensembles $G_{\hat{o}}$, Fund. Math., 16 (1930), 173180 .

13. H. H. Wicke, The non-preservation of $\check{C}$ ech completeness by an open continuous mapping between collectionwise normal $T_{1}$-spaces, Notices of the Amer. Math. Soc., 13 (1966), 601.

14. The regular open continuous images of complete metric spaces, Pacific J. Math., 23 (1967), 621-625.

15. - On the Hausdorff open continuous images of Hausdorff paracompact pspaces, Proc. Amer. Math. Soc., 22 (1969), 136-140.

16. H. H. Wicke and J. M. Worrell, Jr., Open continuous mappings of spaces having bases of countable order, Duke Math. J., 34, No. 2 (1967), 255-272.

17. - On topological completeness of first-countable Hausdorff spaces, submitted for publication.

18. J. M. Worrell, Jr. and H. H. Wicke, Complete $\mu$-spaces, Notices of the Amer. Math. Soc., 14 (1967), 856.

Received July 3, 1968. This work was supported by the United States Atomic Energy Commission.

SANDIA LABORATORIES

Albuquerque, New Mexico 



\section{PACIFIC JOURNAL OF MATHEMATICS}

\section{EDITORS}

H. SAMELSON

Stanford University

Stanford, California 94305

C. R. Новву

University of Washington

Seattle, Washington 98105
J. DUGundJI

Department of Mathematics

University of Southern California

Los Angeles, California 90007

RICHARD ARENS

University of California

Los Angeles, California 90024

\section{ASSOCIATE EDITORS}
E. F. BECKENBACH
B. H. NeumanN
F. WOLF
K. YOSHIDA

\section{SUPPORTING INSTITUTIONS}
UNIVERSITY OF BRITISH COLUMBIA
CALIFORNIA INSTITUTE OF TECHNOLOGY
UNIVERSITY OF CALIFORNIA
MONTANA STATE UNIVERSITY
UNIVERSITY OF NEVADA
NEW MEXICO STATE UNIVERSITY
OREGON STATE UNIVERSITY
UNIVERSITY OF OREGON
OSAKA UNIVERSITY
UNIVERSITY OF SOUTHERN CALIFORNIA
STANFORD UNIVERSITY
UNIVERSITY OF TOKYO
UNIVERSITY OF UTAH
WASHINGTON STATE UNIVERSITY
UNIVERSITY OF WASHINGTON
AMERICAN MATHEMATICAL SOCIETY CHEVRON RESEARCH CORPORATION TRW SYSTEMS
NAVAL WEAPONS CENTER 


\section{Pacific Journal of Mathematics}

\section{Vol. 37, No. $1 \quad$ January, 1971}

Gregory Frank Bachelis and Haskell Paul Rosenthal, On unconditionally

converging series and biorthogonal systems in a Banach space .........

Richard William Beals, On spectral theory and scattering for elliptic

operators with singular potentials .........................

J. Lennart (John) Berggren, Solvable and supersolvable groups in which every element is conjugate to its inverse ........................ 21

Lindsay Nathan Childs, On covering spaces and Galois extensions ..........

William Jay Davis, David William Dean and Ivan Singer, Multipliers and

unconditional convergence of biorthogonal expansions..............

Leroy John Derr, Triangular matrices with the isoclinal property ............

Paul Erdős, Robert James McEliece and Herbert Taylor, Ramsey bounds for

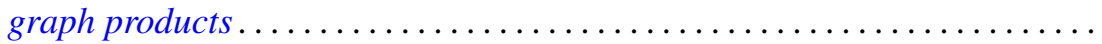

Edward Graham Evans, Jr., On epimorphisms to finitely generated

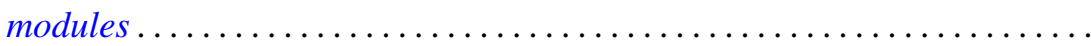

Hector O. Fattorini, The abstract Goursat problem ................. 51

Robert Dutton Fray and David Paul Roselle, Weighted lattice paths .........

Thomas L. Goulding and Augusto H. Ortiz, Structure of semiprime $(p, q)$

radicals ...........................................

E. W. Johnson and J. P. Lediaev, Structure of Noether lattices with join-principal maximal elements ....

David Samuel Kinderlehrer, The regularity of minimal surfaces defined over

slit domains

Alistair H. Lachlan, The transcendental rank of a theory. .

Frank David Lesley, Differentiability of minimal surfaces at the boundary ...

Wolfgang Liebert, Characterization of the endomorphism rings of divisible torsion modules and reduced complete torsion-free modules over complete discrete valuation rings....

Lawrence Carlton Moore, Strictly increasing Riesz norms.

Raymond Moos Redheffer, An inequality for the Hilbert transform ...

James Ted Rogers Jr., Mapping solenoids onto strongly self-entwined,

circle-like continua..........................

Sherman K. Stein, B-sets and planar maps ................... 217

Darrell R. Turnidge, Torsion theories and rings of quotients of Morita

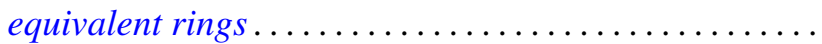

Fred Ustina, The Hausdorff means of double Fourier series and the principle of localization ................................

Stanley Joseph Wertheimer, Quasi-compactness and decompositions for arbitrary relations.

Howard Henry Wicke and John Mays Worrell Jr., On the open continuous images of paracompact $\check{C}$ ech complete spaces... 